\title{
Evaluación psicológica en el ámbito forense: la libertad anticipada en el contexto uruguayo
}

\author{
Psychological assessment in the forensic field: early release in the Uruguayan context
}

Lucía Barboni Pekmezian ${ }^{1}$

Natalia Bonilla Armada ${ }^{2}$

\author{
${ }^{1}$ Departamento de Psicología Social y Organizacional, Facultad de Psicología. Universidad Católica del \\ Uruguay \\ ${ }^{2}$ Instituto Nacional de Criminología, Ministerio del Interior. Uruguay
}

\begin{abstract}
Resumen: Tras la revisión de las condiciones de las cárceles uruguayas y de la reclusión y sus consecuencias, surge un proceso paulatino de cambio en el sistema, que se dirige -desde hace varios años- hacia la promoción del respeto por los derechos humanos de las personas privadas de libertad. Considerando la relevancia de la Psicología aplicada al ámbito jurídico como disciplina que acompasa dicho proceso, el objetivo de esta comunicación es, a partir de los datos que surgen de los informes por libertad anticipada en nuestro país entre 2013 y 2017 y sus características, indagar acerca del rol del psicólogo en este ámbito y los instrumentos de evaluación de mayor uso en las investigaciones en la materia, para así dotar al profesional de criterios diagnósticos mancomunados y a la vez, poder optimizar recursos y resultados. La conclusión se centra en la necesidad de implementar el uso de guías y manuales que garanticen las prácticas profesionales, con herramientas diagnósticas validadas, que permitan al profesional unificar los criterios diagnósticos y optimizar los resultados obtenidos.
\end{abstract}

Palabras clave: peligrosidad, evaluación de riesgo, evaluación forense, pericia psicológica, cárcel

\begin{abstract}
A review of conditions of Uruguayan prisons as well as of imprisonment and its consequences, shows that a gradual process of change in the penitentiary system has begun, aimed at the promotion of respect for human rights of people deprived of their liberty. Given the relevance of applied Psychology in the legal context as a discipline accompanying such process, the aim of this paper is to inquire, based on a documentary review of data arising from early release reports in our country between 2013 and 2017 and characteristics thereof, about the role of psychologists in this matter and about assessment instruments mostly used in research on this issue, in order to provide professionals with joint diagnostic criteria as well as to optimize resources and results. Overall, given the lack of specific formal protocols that guide assessment procedures, there is a need to implement guidelines and protocols that guarantee professional practices as well as to use diagnostic tools that enable the unification of assessment criteria, optimizing results obtained.
\end{abstract}

Keywords: hazard, risk assessment, forensic assessment, forensic psychological reports, prision

Cómo citar esta comunicación:

Barboni Pekmezian, L., \& Bonilla Armada, N. (2018). Evaluación psicológica en el ámbito forense: la libertad anticipada en el contexto uruguayo. Ciencias Psicológicas, 12(2), 285-292.

doi: https://doi.org/10.22235/cp.v12i2.1692

Correspondencia: Lucía Barboni Pekmezian. Comandante Braga 2715, Facultad de Psicología, Universidad Católica del Uruguay; e-mail: lbarboni@ucu.edu.uy. Natalia Bonilla Armada.Instituto Nacional de Criminología. I.N.R. Ministerio del Interior, Montevideo, Uruguay. Agraciada 4129, Montevideo, e-mail: bonillan@vera.com.uy 
Tejero (2016) define la función del "perito", como el ejercicio profesional al emitir un dictamen de valor probatorio, proveniente de los conocimientos propios de su ciencia, con una demanda específica y con el objetivo de auxiliar al juez. En el Código General del Proceso uruguayo, se procede a la prueba pericial cuando son necesarios conocimientos científicos, artísticos o técnicos especiales (Ley No 15982; 1988).

Se define el peritaje psicológico forense como un instrumento de asesoría a la autoridad judicial a través de un dictamen basado en una evaluación en profundidad en un área específica y con un objetivo puntual (Ching, 2005). Si bien es importante destacar que el informe pericial no es vinculante para el jurista, se trata de un elemento auxiliar de alta relevancia para el Juez (Tejero, 2016).

A los efectos de contar con ciertos parámetros de procedimiento en la evaluación pericial, se cuenta con el Manual elaborado por los peritos psicólogos del departamento Médico-Criminológico del Instituto Técnico Forense del Poder Judicial de Uruguay (de aquí en adelante I.T.F.). Éste enuncia que el profesional debe considerar ciertas características al momento de periciar una persona como: la ausencia de voluntariedad absoluta al momento de someterse a una evaluación, el stress causado por el proceso judicial, y la necesidad del sujeto de presentarse positivamente, aumentando ello la deseabilidad y el margen de error en las pruebas (Instituto Técnico Forense, 1998; Muñoz, 2013; Echeburúa, Muñoz, \& Loinaz, 2011).

Los peritos psicólogos en el contexto uruguayo, asesoran en la administración de la justicia penal en los diferentes momentos que atraviesa el proceso, desde las etapas iniciales de comprobación del los hechos criminales, hasta la resolución de la posible concesión de la libertad anticipada.

Si bien el instrumento fundamental y más utilizado en el proceso de evaluación es la entrevista pericial semi-estructurada, el profesional debe contar con otras herramientas para reducir la incidencia de la variable subjetiva, apuntando a lograr conclusiones objetivas en la resolución de las evaluaciones.

En el cometido de planificar y ejecutar una evaluación cuyo objetivo es responder respecto a la presencia de los posibles factores de riesgo y/o de protección estáticos y dinámicos (Arbach et al.,
2015) en el marco de las implicancias que supone el acceso al beneficio de la libertad anticipada, es obligatorio aproximarse a lo que establece la normativa. El Instituto Nacional de Criminología (de aquí en adelante I.NA.CRI.), funciona hasta el día de la fecha dentro del Instituto Nacional de Rehabilitación (de aquí en adelante I.N.R.). Dentro de su Centro Nacional de Dictámenes Criminológicos se realizan -entre otras- las evaluaciones de la población privada de libertad que solicita su Libertad Anticipada por el Art. 328, del Código del Proceso Penal, (de aquí en adelante C.P.P.). Se trata de un trámite que obligatoriamente (hasta 31 de Octubre de 2017) debía contar con la pericia realizada exclusivamente por los técnicos del Instituto, y de la misma, surgía una evaluación de Personalidad y Peligrosidad (Instituto Técnico Forense, 1998) con la finalidad de estudiar el funcionamiento psico-social actual del sujeto en pro de la recomendación o no a dicho beneficio. La concesión o la negación de la misma, tratándose de un dictamen psicológico no vinculante, era competencia de la Suprema Corte de Justicia (de aquí en adelante S.C.J.).

Previo a la reciente reforma, el C.P.P. establecía en su artículo 328 que la S.C.J. podrá conceder la libertad anticipada en los siguientes casos: si la condena es de penitenciaría y el penado ha cumplido la mitad de la pena impuesta; si la pena es de prisión o multa, sea cual fuese el tiempo de reclusión sufrida; si el penado ha cumplido las dos terceras partes de la pena impuesta. Sólo podría negarla, por resolución fundada, en los casos en que los signos de rehabilitación del condenado no sean manifiestos. En todos los casos debería contar con el informe preceptivo realizado por el I.NA.CRI.

Sin embargo, el C.P.P. tuvo una nueva redacción introducida por la Ley $\mathrm{N}^{\circ} 19.293$ (2015) donde se prevé que el beneficio de la libertad anticipada podrá otorgarse a los sujetos penados, que estando privados de libertad, se les pueda formular un pronóstico favorable de reinserción social teniendo en cuenta su conducta, personalidad, forma y condiciones de vida. Se considera que: si la pena recaída fuera de prisión, podrá solicitarla en cualquier momento; si la condena fue de penitenciaría, podrá solicitarla habiendo cumplido la mitad de la pena; en caso de que a la pena de penitenciaría se le haya adicionado medidas de seguridad eliminativas, se le podrá solicitar luego de que haya alcanzado las dos 
terceras partes de la pena. Esta modificación de la Ley suplanta la pericia por el informe técnico referido a las aptitudes de resocialización del penado, dejando de lado la competencia exclusiva del I.NA.CRI en la evaluación para la Libertad Anticipada (Art. 229; Ley No 19293, 2015). Posteriormente el juez de Ejecución y Vigilancia resolverá previa vista del Ministerio Público, disponiendo de: el informe de conducta carcelaria y los informes técnicos que se dispongan referidos a las aptitudes de resocialización del penado; la reliquidación de pena por trabajo y estudio, y la planilla de antecedentes penales actualizada. De esta forma se busca modificar un trámite que hasta el momento parecía ser muy extenso ya que no solo se suprime la evaluación pericial realizada por el I.NA.CRI., sino que ya no será la S.C.J. la que resolverá si otorga o no la libertad anticipada.

El comportamiento violento ha sido considerado como uno de los elementos más característicos de la delincuencia grave, y asociado a ello, la atribución de peligrosidad a quienes han cometido delitos violentos ha sido utilizada como argumento explicativo y predictivo, tanto de la gravedad de los actos delictivos, como de la reincidencia (Andrés-Pueyo \& Arbach, 2014; Andrés-Pueyo \& Redondo, 2007), cuestiones directamente vinculadas con el punto de pericia de libertad anticipada.

El históricamente debatido concepto de "peligrosidad", hace referencia a la propensión que tiene el individuo de cometer actos peligrosos y violentos, pretendiendo resumir con una aparente claridad la idea de ser por excelencia el predictor de la conducta violenta futura (Scotto \& Resnick, 2006). Supone una cualidad estática, una disposición psicológica (Arbach \& Andrés-Pueyo, 2007) que podría resultar altamente estigmatizante para el sujeto evaluado, tratándose de un juicio clínico llevado a cabo de forma idiosincrática y con deficiencias en su conceptualización (Arbach et al., 2015). Dado que el desarrollo de la psicología criminológica ha demostrado la ineficaz y limitada capacidad predictiva que ha tenido el concepto de peligrosidad para tomar decisiones prospectivas en contextos clínicos, forenses o penitenciarios se ha promovido a nivel internacional la utilización del concepto evaluación de riesgo (Andrews \& Bonta, 2003).

La valoración del riesgo de conducta violenta como alternativa al diagnóstico de peligrosidad (como predictor de la conducta violenta), estable- ce que cada tipo de violencia tiene sus factores de riesgo y de protección particulares (Andrés-Pueyo \& Echeburúa, 2010) y que la forma en que se realiza la evaluación del comportamiento violento y la planificación en la intervención, tiene un impacto directo en las decisiones judiciales, en el bienestar social y en la labor profesional (OchoaBalarezo et al., 2017).

Tanto desde ámbitos formales, como informales, hay un interés social por conocer y predecir el comportamiento violento de las personas, pero además, surge la necesidad de sistematizar criterios con base empírica, para llevar a cabo dichas predicciones (Folino \& Escobar Córdoba, 2004). Determinados caracteres psicológicos individuales (rasgos de personalidad y habilidades psicosociales) son considerados factores de riesgo que inciden en el comportamiento violento predisponiendo la ejecución de conductas antisociales. Al combinarse estas conductas con determinados factores sociales, originan manifestaciones conductuales violentas graves o extremas. Conocer los mecanismos de acción de los factores de riesgo, de los factores desencadenantes y la interacción de ambos (oportunidad, contexto, características del caso), es lo que permite predecir y prevenir el comportamiento violento (Andrés-Pueyo \& Redondo, s.f; Loinaz, 2017) lo cual resulta especialmente útil para las evaluaciones por libertad anticipada.

La creación de múltiples guías de valoración de riesgo de conducta violenta en las últimas décadas y la creciente adaptación de las mismas a nivel internacional da cuenta de los cambios que se vienen desarrollando en los sistemas de evaluación. Las investigaciones a nivel internacional ponen de manifiesto la preocupación por sistematizar la evaluación del riesgo de violencia y en consecuencia, la tarea de planificar y monitorear la intervención destinada a prevenir la reincidencia de estas conductas (Ochoa-Balarezo et al., 2017).

La utilización de instrumentos de valoración de riesgo se ha convertido en un procedimiento habitual de administración para los psicólogos de Reino Unido, Australia, Estados Unidos y Dinamarca; sin embargo, hay una mayor tendencia hacia las herramientas del tipo actuarial, que a las de juicio clínico estructurado (Singh et al., 2014). Dichos instrumentos facilitan la recepción y revisión crítica de los actores del sistema, otorgando transparencia a los procesos, tanto de evaluación, 
como de toma de decisiones (Muñoz \& LópezOssorio, 2016).

Dentro de los nueve instrumentos más utilizados que surgen de una encuesta (Viljoen, McLachlan, \& Vincent, 2010) se encuentran: Assessing Risk for Violence-HCR-20 (Douglas et al., 2013) Sexual Violent Risk-SVR-20 (Boer et al., 1998), Spousal Assault Risk Assessment Guide-SARA (Kropp \& Hart, 2000) y Structured Assessment of Violence Risk in Youth -SAVRY (Borum, Bartel, \& Forth, 2003); Level of Service Inventory-revised- LSI-R (Andrews \& Bonta, 1995), the Hare Psychopathy Checklist- revisedPCL-R (Hare, 2003), Sex Offender Risk Appraisal Guide- SORAG (Quinsey et al., 1998), Static-99 (Hanson \& Thornton, 1999) y Violence Risk Appraisal Guide- VRAG (Harris et al., 1993).

Sobre los mismos hay un importante acervo de investigaciones que permiten visualizar la importancia de su inclusión en los nuevos procedimientos (Viljoen et al., 2010; Gacono, 2000; Arbach et al., 2015; Fazel et al., 2012; Loinaz, 2017). Si bien el panorama en Latinoamérica evidencia que las herramientas de valoración de riesgo se encuentran en un incipiente desarrollo, aun prima la utilización de otro tipo de instrumentos (Singh, Condemarín, \& Folino, 2013; Arbach et al., 2017).

Esta comunicación tiene como objetivo aproximarnos a la situación de la pericia psicológica forense en personas que solicitaron el beneficio de la libertad anticipada, a partir de un estudio documental de los registros nacionales.

\section{Estudio Documental}

En este estudio documental se recabó información a partir de los registros que conforman la base de datos de I.NA.CRI., entre los años 20132017. En los mismos se indagaron los siguientes asuntos:

- Cantidad de personas periciadas anualmente

- Tipo de delito que surge en la carátula del expediente

- Unidad penitenciaria en la que fue evaluada la persona privada de libertad

- Formación profesional del Técnico de I.NA. CRI

- Tipo de recomendación del Técnico de I.NA. CRI

Una vez obtenidos estos datos, se procedió a analizar las Resoluciones de la S.C.J publicadas en la sección de Estadísticas de la página web del Poder Judicial (Poder Judicial, 2017) en relación con cuántas libertades anticipadas fueron otorgadas y cuántas negadas, en forma genérica.

\section{Resultados}

En base al estudio documental realizado sobre los registros de personas que fueron periciadas psicológicamente ante la solicitud de libertad anticipada en el marco del proceso penal de Uruguay, se presentan los resultados que parten de la revisión de los registros que recoge el sistema de control de expedientes y pericias realizadas del I.NA.CRI, como las resoluciones que toma en los mismos periodos la S.C.J. (Poder Judicial, 2017) respecto a la concesión de libertades anticipadas entre los años 2013 y 2017.

La figura 1 muestra la totalidad de pericias anualizadas con recomendación favorable (informes positivos) y desfavorable (informes negativos) para obtener el beneficio por I.NA.CRI. En relación a las resoluciones de la S.C.J. ${ }^{1}$, figuran la totalidad de concesiones y denegaciones de libertad anticipada también de forma anualizada.

En el año 2013, según los dictámenes realizados por los técnicos, se recomendó acceder a 736 casos a la libertad anticipada, mientras que a 701 casos se les aconsejó no acceder al beneficio. En este mismo año la S.C.J. concedió 554, y negó 961 libertades anticipadas.

En el año 2014 se registran 915 dictámenes con recomendación positiva, mientras que, en contrapartida, en 727 casos se recomienda no acceder a la libertad anticipada. La S.C.J. concedió 728 libertades anticipadas y negó 1078 .

Prosiguiendo, en el año 2015, surgen 828

1 La SCJ refiere ciertos casos en la categoría de "No está en estado", que hace referencia a aquellas solicitudes que fueron tramitadas erróneamente, por ejemplo no contaban con los requisitos para solicitar dicho beneficio, o directamente no era este beneficio que estaba solicitando, ni ninguno de los beneficios tramitados por la Sección Libertades. Existen 58 casos en 2013, 54 en 2014, 57 en 2015, 25 en 2016, 12 en 2017.

Se identifican "Casos no resueltos" en INACRI que hacen referencia a resultados que no fueron positivos ni negativos, ya que se presentaron distintos motivos, por ejemplo; que el interno fue entrevistado por error; no se encontró en el establecimiento al recluso; el mismo se encuentra fugado; no quiere concurrir; falleció; fue liberado; se niega a la entrevista; o, le falta solicitud del derecho; no cumple con la $1 / 2$ de la pena, entre otros aspectos. Desde los datos, se desconoce qué ocurrió posteriormente con estas personas, es decir no se ha logrado saber si luego fueron entrevistadas. Existen 123 casos en 2013, 202 en 2014, 324 en 2015 y 237 en 2017. No contamos con los datos de 2016. 


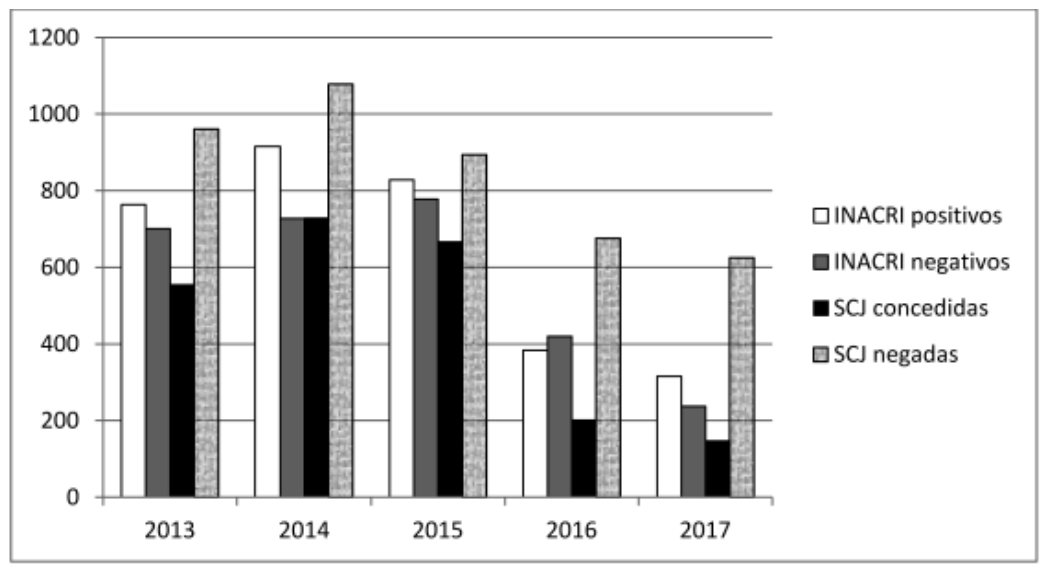

Figura 1

Totalidad de informes y resoluciones entre 2013 y 2017

recomendaciones favorables, en cambio 778 recomendaciones fueron negativas. En esta oportunidad, se concedieron 666 libertades anticipadas por parte de la S.C.J., y se negaron 894.

Desde el año 2013 al año 2015 se efectuaron más recomendaciones positivas respecto a dicho beneficio, que negativas. Sin embargo, se logra visualizar que la situación cambia en el año 2016, debido a que se constata que hubo 420 informes negativos y 384 positivos. En este mismo año la S.C.J. concedió 201 libertades y negó 676.

Finalmente, en el año 2017, la S.C.J. concedió 147 libertades anticipadas y negó 625, mientras que I.NA.CRI. recomendó positivamente 316 y negativamente 237 .

\section{Discusión}

El estudio tuvo como objetivo describir las particularidades y procedimientos inherentes al rol del psicólogo como asesor judicial en las solicitudes de libertad anticipada en Uruguay a través de una revisión teórica de la temática y la presentación de las cifras referentes a las pericias realizadas entre 2013 y 2017 desde las cuales se problematiza la necesidad de una revisión en las prácticas profesionales acorde a lo que las publicaciones internacionales recomiendan respecto a la evaluación psicológica-forense. La intención primordial ha sido presentar por primera vez información relativa al panorama uruguayo en pericias psicológicas por libertad anticipada.

Respecto a los hallazgos preliminares del presente estudio documental, del registro de pericias realizadas se detecta la necesidad de informatizar con mejor calidad los informes, se visualiza un descenso en la cantidad de pericias y resoluciones realizadas que responde -entre otras cosas- a la necesidad de más recursos humanos, así como también la falta de guías que orienten las prácticas profesionales. Además surge que salvo casos excepcionales, la única técnica utilizada ha sido la entrevista. Si bien se sostiene que la administración de tests y otras técnicas de evaluación en pro de procurar mayor objetividad debe ser cautelosa, y se apela a una planificación que considere tanto su utilidad, tiempo, calidad científica y limitaciones, los instrumentos de evaluación de riesgo en el ámbito pericial, son ventajosos en múltiples sentidos (Muñoz \& López Ossorio, 2016).

Del relevamiento documental realizado se detectó la ausencia de criterios que unifiquen conceptualizaciones como son la rehabilitación, la progresividad, el proceso de resocialización o la vulnerabilidad frente al delito. Además se puede observar la mínima utilización de instrumentos de evaluación específicos del área forense teniendo en cuenta las particularidades de la población evaluada. También la dificultad con la que se enfrenta diariamente el técnico al evaluar un plan de egreso (como factor de riesgo y como factor de protección) con la ausencia en el evaluado de redes sociales y posibilidad de entrar en el mercado laboral de forma estable y formal al momento del egreso. Y fundamentalmente, la rigurosidad que requiere el evidenciar una posible relación entre una estructura de personalidad y un pronóstico criminológico. 
Desde Febrero de 2013 a Diciembre de 2017, la S.C.J. otorgó 2296 libertades anticipadas, habiendo un total de 3179 recomendaciones favorables y 2863 desfavorables por parte de I.NA.CRI. No se corresponden en el periodo la cantidad de evaluaciones realizadas con la cantidad de resoluciones que expide la S.C.J, posiblemente por un desfasaje en el tiempo de trámite, lo cual supone una debilidad al momento de analizar la correlación entre los datos. Tratándose de una resolución que implica el acceso del sujeto a su libertad ambulatoria de forma anticipada al cumplimiento del total de su pena, considerándose las diferentes repercusiones que ello tiene (fundamentalmente respecto a los delitos más violentos), y que en la mayoría de casos (exceptuando 2017) respecto a la totalidad de informes positivos, se ha fallado a favor de la misma, se valora de especial relevancia la calidad científica de las pericias que estarán siendo de valor probatorio para la S.C.J.

Considerando la complejidad de la función pericial; la ausencia de fuentes bibliográficas locales y con la consulta de informantes calificados, se perfila un panorama donde se destaca que I.NA.CRI. no cuenta a la fecha con una guía de práctica profesional formal y de difusión pública que permita orientar, sugerir y protocolizar el paso a paso de las evaluaciones psicológicasforenses. Sin embargo, en el ámbito de la psicología forense a nivel internacional y en diferentes áreas, existen guías orientadoras para los profesionales de significativa implicancia y especialmente recomendadas (Colegio Oficial de Psicólogos de Madrid, 2009; Asociación de Psicólogos Forenses de la Administración de Justicia, 2018; American Psychological Association, 2013; Colegio Oficial de Psicología de Catalunya, 2014; Instituto Nacional de Medicina Legal y Ciencias Forenses, 2010; Fiscalía de Chile, 2008; Ruiz, 2014; Instituto de Medicina Legal y Ciencias Forenses, 2016; Poder Judicial, 2008).

La creación de guías de prácticas profesionales, la implementación de herramientas de evaluación forense adaptadas a nuestra población que le permitan al profesional unificar los criterios de evaluación y optimizar los resultados obtenidos, una mejor sistematización de datos, y la promoción de investigación en el ámbito, son ajustes necesarios para seguir avanzando en el desarrollo del área.
Una de las principales revisiones que debemos destacar a las prácticas actuales, es la urgencia que supone entender que el rol pericial desde su propia conceptualización indica la especialización en un área y ello obliga a que los profesionales que ejercen tal función tengan la formación específica que atiende a las particularidades de la rama.

Trabajar desde la valoración del riesgo de conducta violenta en lugar de estimar grados de peligrosidad, supone, no solamente un avance en la rigurosidad del trabajo pericial, sino una forma de pensar en cómo gestionar ese riesgo considerando que el mismo es dinámico, situacional, en definitiva, contemplar que son diversos los factores de riesgo que estarían incidiendo más allá de los individuales. Aun así, se destaca la importancia de que los técnicos conozcan las herramientas y sepan seleccionar la adecuada para cada caso, atendiendo especialmente a la validez predictiva de las mismas, pero además la precisión que requiere definir qué es lo que va a evaluarse, para qué y cómo.

La aparente transición hacia un panorama más óptimo en las prácticas latinoamericanas (Singh, Condemarín, \& Folino, 2013) debe ser un incentivo al momento de seguir investigando y Uruguay no puede quedar afuera en este desafío.

\section{Referencias}

American Psychological Association (2013). Specialty Guidelines for Forensic Psychology. American Psychologist. 68 (1), 7-19. DOI: 10.1037/a0029889

Andrés-Pueyo, A. \& Arbach, K. (2014). Peligrosidad y valoración del riesgo de violencia en contextos forenses. En E. García-López. (Ed.), Psicopatología Forense: comportamiento humano y tribunales de justicia. (pp. 505-525). Bogotá: Editorial El Manual Moderno Colombia. Recuperado de https://ebookcentral.proquest. com/lib/ucusp/detail.action?docID $=3223956$

Andrés-Pueyo, A. \& Echeburúa, E. (2010). Valoración del riesgo de violencia: instrumentos disponibles e indicaciones de aplicación. Psicothema, 22, 403-409. Recuperado de http://www.psicothema.com/PDF/3744.pdf

Andrés-Pueyo, A. \& Redondo, S. s.f Aportaciones psicológicas a la predicción de la conducta violenta: reflexiones y estado de la cuestión. Departamento de personalidad. Grupo de Estudios Avanzados en Violencia (GEAV). Universidad de Barcelona. España. Recuperado de http:// www.ub.es/personal/geav.htm

Andrés-Pueyo, A. \& Redondo, S. (2007). La predicción de la violencia: entre la peligrosidad y la valoración del riesgo de violencia. Papeles del Psicólogo, 28, 157-173. Recuperado de http://www.redalyc.org/ pdf/778/77828301.pdf

Andrews, D., Bonta, J. (1995). LSI-R: The Level of Service Inventory-Revised. Toronto, ON: Multi-Health Systems Inc. 
Andrews, J., Bonta, R. (2003). The psychology of criminal conduct (3rd. Ed.). Cincinnati: Anderson Pub. Cgo.

Arbach, K. \& Andrés-Pueyo, A (2007) Valoración del riesgo de Violencia en enfermos mentales con el HCR-20 Papeles del Psicólogo, 2007. Vol. 28(3), pp. 174-186 Recuperado de http://www.redalyc.org/ pdf/778/77828301.pdf

Arbach, K., Dezmarais, S. L., Hurducas, C., Condemarin, C., Dean, K., Doyle, M., Folino, J.O. ... Singh, J.P. (2015). La práctica de la evaluación de riesgo en España. Revista de Facultad de Medicina, 63(3), 357366. DOI: http://dx.doi.org/10.15446/revfacmed. v63n3.48225

Arbach, K., Bondaruk, A., Carubelli, S., Palma-Vegar, M. F., \& Singh, J. P. (2017). Evaluación forense de la peligrosidad: Una aproximación a las prácticas profesionales en Latinoamérica. Revista Latinoamericana de Ciencia Psicológica, 9(1), 1-15. DOI: https:// dx.doi.org/10.5872/psiencia/9.1.23

Asociación de Psicólogos Forenses de la Administración de Justicia (2018). Evaluación psicológica forense de los abusos y maltratos a niños, niñas y adolescentes. Guía de buenas prácticas. España. Recuperado de http://copmelilla.org/descargas/pdf/uiebuenaspracticasymaltratoinfantil.pdf

Boer, D., Hart, S., Kropp, P., \& Webster, C. (1998). Manual for the Sexual Violence Risk-20. Lutz, Florida: Psychological Assessment Resources, Inc.

Borum, R., Bartel, P., \& Forth, A. (2003). Manual for the Structured Assessment for Violence Risk in Youth (SAVRY). Odessa, FL: Psychological Assessment Resources.

Ching, R. (2005). Psicología Forense: Principios fundamentales. Recuperado de https://books.google.com. uy/books?id=bSd3q_EuXW0C\&printsec $=$ frontcov er\&dq $=$ Ching, + R. $+(2005) .+$ Psicolog $\% \mathrm{C} 3 \% \mathrm{ADa}+\mathrm{F}$ orense:+Principios+fundamentales. + (Primera+reim presi $\%$ C3\%B3n).+San+Jos\%C3\%A9,+Costa+Rica: + Editorial+Universidad+Estatal+a+Distancia.\&hl= es $419 \& \mathrm{sa}=\mathrm{X} \&$ redir_esc $=\mathrm{y} \# \mathrm{v}=$ onepage $\& \mathrm{q} \& \mathrm{f}=$ false

Colegio Oficial de Psicólogos de Madrid (2009). Guía de buenas prácticas para la elaboración de informes psicológicos periciales sobre custodia y régimen de visitas de menores. Madrid. Recuperado de http:// www.copmadrid.org/webcopm/recursos/guia_buenas_practicas_informes_custodia_y_regimen_visitas julio2009.

Colegio Oficial de Psicología de Catalunya (2014). Guía de buenas prácticas para la evaluación psicológica forense y la práctica pericial. Catalunya. Recuperado de http://www.infocop.es/pdf/guiaforense2014.pdf .

Douglas, K. S., Hart, S. D., Webster, C. D., \& Belfrage, H. (2013). HCR-20 (Version 3): Assessing Risk for Violence. Burnaby, BC, Canada: Mental Health, Law, and Policy Institute, Simon Fraser University.

Echeburúa, E., Muñoz, J.M., \& Loinaz, I. (2011). La evaluación psicológica forense frente a la evaluación clínica: propuestas y retos de futuro. International Journal of Clinic and Health Psychology, 11 (1), 141-159. Recuperado de http://www.redalyc.org/ pdf $/ 337 / 33715423009$.pdf

Fazel, S., Singh, J., Doll, H, \& Grann, M. (2012). Use of risk assessment instruments to predict violence and antisocial behaviour in 73 samples involving 24827 people. Systematic review and meta-analysis. British Medical Journal, 24 (345). DOI: https://doi.org/10.1136/bmj. e4692
Fiscalía de Chile (2008). Evaluación pericial psicológica de credibilidad de testimonio. Documento de trabajo interinstitucional. Santiago de Chile. Recuperado de http://www.fiscaliadechile.cl/Fiscalia/ archivo $\mathrm{id}=625 \& \mathrm{pid}=60 \& \mathrm{tid}=1$

Folino, J.O., \& Escobar Córdoba, F. (2004). Nuevos aportes a la evaluación de riesgo de violencia. MedUnab. 7 (20), 99-105. Recuperado de https://revistas.unab.edu. co/index.php/medunab/article/view/227/210

Gacono, C. (2000). The clinical and forensic assessment of Psychopathy: A practitioner's guide. Mahwah, NJ: Lawrence Erlbaum

Hanson, R. K., \& Thornton, D. (1999). Static-99: Improving actuarial risk assessments for sex offenders. User Report 99-02. Ottawa: Department of the Solicitor General of Canada.

Hare R. (2003). The Hare Psychopathy Checklist - Revised Manual. 2nd ed. Toronto: Multi-Health Systems, Inc.

Harris, G.T., Rice, M.E., \& Quinsey, V.L. (1993). Violent recidivism of mentally disorders offenders: the development of a statistical prediction instrument. Criminal Justice and Behaviour, 20, 315-335. https:// doi.org/10.1177/0093854893020004001

Instituto Nacional de Medicina Legal y Ciencias Forenses (2010). Guía para la realización de pericias psiquiátricas y psicológicas forenses mediante autopsia psicológica en la determinación de la manera de muerte (suicida, homicida o accidental). Bogotá. Recuperado de http://www.medicinalegal.gov.co/ documents/20143/40473/Gu\%C3\%ADa+para+la+rea lizaci $\% \mathrm{C} 3 \% \mathrm{~B} 3 \mathrm{n}+\mathrm{de}+$ pericias + psiqui $\% \mathrm{C} 3 \% \mathrm{~A} 1$ tricas $+\mathrm{o}+$ psicol $\% \mathrm{C} 3 \% \mathrm{~B} 3$ gicas + forenses + mediante+autops $\mathrm{ia}+\mathrm{psicol} \% \mathrm{C} 3 \% \mathrm{~B} 3$ gica + en $+\mathrm{la}+$ determinaci $\% \mathrm{C} 3 \% \mathrm{~B} 3$ $\mathrm{n}+\mathrm{de}+\mathrm{la}+$ manera + de + muerte + suicida $\% 2 \mathrm{C}+$ homida + pdf/3e166326-5933-7734-5b32-a80171456ac3

Instituto de Medicina Legal y Ciencias Forenses (2016) Guía de evaluación psicológica forense en casos de violencia contra las mujeres y los integrantes del grupo familiar; y en otros casos de violencia. Lima. Recuperado de https://portal.mpfn.gob.pe/descargas/ Guia 04.pdf

Instituto técnico Forense, (1998). Poder Judicial del Uruguay. Manual del Instituto Técnico Forense. Montevideo.

Kropp,P.R. \& Hart, S. D. (2000). The Spousal Assault Risk Assessment (SARA) Guide: Reliability and validity in adult male offenders. Law and $\mathrm{Hu}$ man Behaviour, 24, 101-118. DOI: http://dx.doi. org/10.1023/A:1005430904495

Ley No 15982 . Código General del Proceso. Uruguay, 14 de noviembre de 1988. Recuperado de https://www.impo. com.uy/bases/codigo-general-proceso/15982-1988

Ley No 19.293. Código del Proceso Penal. Uruguay, 9 de enero de 2015. Recuperado de https://legislativo. parlamento.gub.uy/temporales/leytemp6967303.htm

Loinaz, I. (2017). Manual de evaluación del riesgo de violencia. Madrid, España: Ediciones Pirámide.

Muñoz, J.M. (2013). La evaluación psicológica forense del daño psíquico: propuesta de un protocolo de actuación pericial. Anuario de Psicología Jurídica, 23, 61-69. Recuperado de http://www.copmadrid.org/webcopm/ publicaciones/juridica/jr2013v23a10.pdf

Muñoz, J. M., \& López- Ossorio, J.J. (2016). Valoración psicológica del riesgo de violencia: alcance y limitaciones para su uso en el contexto forense. Anuario de Psicología Jurídica, 26, 130-140. DOI: https://doi. org/10.1016/j.apj.2016.04.005 
Ochoa-Balarezo, J.V., Guillén, X.K., Ullauri-Ortega, Narváez, J.L., León-Mayer, E., \& Folino, J. (2017) Sistematización de la evaluación de riesgo de violencia con instrumentos de juicio profesional estructurado en Cuenca, Ecuador. Maskana, 9(1), 1-14. DOI: https://doi.org/10.18537/mskn.08.01.01

Poder Judicial (2008). Evaluación y recomendaciones para la elaboración de peritajes psicológicos y psiquiátricos en el Poder Judicial. San José, Costa Rica. Recuperado de https://www.poder-judicial.go.cr/violenciaintrafamiliar/index.php/de-su-interes?download=3 84:peritajespsicolpsiquiatpj

Poder Judicial (2017). Estadísticas. Sección Libertades y Visitas de Cárceles (enero a diciembre de 2017). Uruguay. Recuperado de http://poderjudicial.gub. uy/images/Estad\%C3\%ADsticas_Enero_a_diciembre_2017.pdf

Quinsey, V. L., Harris, G. T., Rice, M. E., \& Cormier, C. A. (1998). Violent offenders: Appraising and managing risk. Washington, DC: American Psychological Association.

uiz, J.I. (Ed.) (2014). Manual para la formulación, diseño $y$ evaluación de programas penitenciarios. Bogotá: Ministerio de Justicia y del Derecho, Instituto Nacional Penitenciario y Carcelario-INPEC y Universidad Nacional de Colombia, Laboratorio de Psicología Jurídica.
Scott, C. L., \& Resnick, P. J. (2006). Violence risk assessment in persons with mental illness. Aggression and Violent Behaviour, 11 (6), 598-611. Doi: http://dx.doi. org/10.1016/j.avb.2005.12.003

Singh, J. P., Desmarais, S. L., Hurducas, C., Arbach, K., Condemarin, C., Dean, K., Doyle, M. ... Otto, R. K. (2014). International perspectives on the practical application of violence risk assessment: a global survey of 44 countries. International Journal of Forensic Mental Health, 13(3), 193-206. DOI: https://dx.doi.or g/10.1080/14999013.2014.922141

Singh, J. P., Condemarín, C., \& Folino, J. (2013). El uso de instrumentos de evaluación de riesgo de violencia en Argentina y Chile. Revista Criminalidad, 55(3), 279290. Recuperado de http://www.scielo.org.co/pdf/ crim/v55n3/v55n3a06.pdf

Tejero, R. (2016). Ejercicio profesional del psicólogo forense y pautas para el orientador. TSOP: Psicología forense y justicia social: estrategias de intervención. X, 10-24. Recuperado de https://ebookcentral.proquest.com/lib/ ucusp/reader.action?docID $=4823934$

Viljoen JL, McLachlan K, \& Vincent GM (2010). Assessing violence risk and psychopathy in juvenile and adult offenders: a survey of clinical practices. Assessment, 17, 377-95. DOI: https://doi. org/10.1177/1073191109359587 